\title{
Editorial: Random dot stereoscopic slides
}

Fascinating issues are raised by the paper by Archer, Miller, Helveston, and Ellis in this number of the $B J O$. I have always wondered how genuine the oftquoted mechanisms concerned with binocular and stereoscopic vision really are. Disparate images, Panum's area, diplopia avoidance, all have a somewhat unsatisfying and rather negative quality as explanations for the brilliant mechanism of binocular stereoscopic vision. The whole business of using both eyes together is mysterious: almost everything is "diplopic" around us except our "object of regard," and yet we do not seem to notice it. Admittedly we can make it rather obvious, for example by holding up a pencil while looking at the television, when the pencil will appear double. Interestingly enough, if the pencil is fixated the "doubleness" of the picture on the television is not nearly so obvious unless captions appear. Thus there seem to be two sorts of physiological diplopia - the usual "unnoticed" type and the more contrived "noticed" type, where a solitary object is deliberately singled out for attention but not fixation.

Since therefore that we accept so much diplopia in everyday life with equanimity, it is perhaps a little surprising that when "true" diplopia occurs it is so catastrophically different from the so-called "physiological" variety. The difference must surely lie in the interpretative interaction between eye and brain, in which the search for meaning, for evidence of reality, for correspondence with other experiences, for a good fit with the previously perceived facts of life, is relentlessly pursued by our senses and our brain acting in concert. Thus our continual visual "surveillance" of our environment seems to involve a series of extremely rapidly repeated judgments.

We tend to reject the impossible (two different objects in the same place at the same time, so-called confusion), the apparently absurd (true diplopia), or the chaotic (the random dots) and to hold on for as long as possible to any image which appears to have order and meaning. However, since diplopia is so commonly seen as a physiological phenomenon, as mentioned above, it is not altogether surprising that its avoidance is not necessarily central to the maintenance of fusion, as Archer and colleagues have now shown.

I have always thought of stereoscopic vision as a form of rationalisation. First we look at the cube slightly from the right, then slightly from the left, and after a bit (? a few milliseconds) the penny drops that it is a cube, in other words it is stereoscopic. Actually, you can do this almost as well with one eye if you move your head from side to side. You can even do it to some extent when viewing an optic disc with a direct ophthalmoscope and can immediately obtain a weak stereoscopic effect. Perhaps "monocular weak stereopsis" would be a better term that the somewhat infelicitous mouthful "parallax." My dictionary defines it as "apparent displacement of an object, caused by actual change of the point of observation." However, this definition would apply only to a theoretical point object. A lot more happens to a real object. For example, the side of our previously mentioned cube actually changes shape or, given a big swing of the head, may even disappear completely.

Thus the somewhat unsatisfactory explanations about stereopsis depending on "disparate images" and fusional reserve being used to "avoid diplopia" have hitherto seemed a bit thin, so it is most exciting to get some new information from Archer and his colleagues strengthening the "order out of chaos" philosophy.
RJHS 